\title{
Is this the bionic man?
}

\author{
Systems that allow a brain to control a computer are inching ever closer to reality - but their most \\ important applications may be different from those envisaged by science fiction.
}

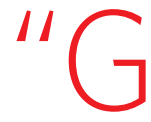

entlemen, we can rebuild him. We have the technology," announced the narrator at the start of the 1970s television series The Six Million Dollar Man. The programme showed scientists reconstructing the shattered body of crash victim Steve Austin with bionic implants he could control with his mind. At the time, this was pure fantasy, but two papers in this week's Nature suggest that the direct interfacing of the human brain with computers or robots is no longer confined to fiction.

Both papers report the development of electronic brain implants, called neuroprostheses, that can translate the intention to move into the actual movement of a robotic device, or of a cursor on a computer screen. The hope is to give paralysed patients greater ability to interact with their environments and perhaps, ultimately, to bypass damaged spinal cords and restore movement to lifeless limbs.

The papers represent a culmination of decades of investigation by many research groups into computing, engineering and the neurobiology of animals and humans. Yet they represent a technology that is still in its infancy: much remains to be done to make such neuroprostheses a clinical reality.

On page 164, researchers led by John Donoghue of Brown University in Rhode Island describe how they helped a 25-year-old patient whose spinal cord had been severed. They implanted an array of electrodes into the area of his brain that controls movement, the motor cortex, and connected them to a computer interface. Even though his motor cortex had been deprived of its normal interaction with the rest of his nervous system for three years, the patient was able to use the system to control a computer cursor, letting him open e-mail, control a television, and move objects using a robotic arm.

\section{Rapid progress}

Donoghue's team is not the first to experiment with such implants. However, previous attempts have succeeded only in getting patients to move a cursor horizontally. Other less-invasive techniques, including ones that use scalp electrodes to pick up brain activity, have taken months of training to adapt for use. And techniques that rely on eye movements have the disadvantage that operating them requires a patient's complete attention. In Donoghue's experiment, the patient adapted to the system within minutes, and was able to converse while making use of it.

But this type of neuroprosthetic system can be rather slow to use. So a group led by Krishna Shenoy of Stanford University, working with monkeys that are not paralysed, has established a technique to speed up the interface of brain and machine (see page 195).

The progress that has been made is remarkable, but many obstacles must still be overcome. The current prosthesis requires the patient to be tethered to a bulky cart of equipment, and it needs constant fine-tuning by a team of technicians. The prototype implant has wires that penetrate the skull and skin, but this carries a risk of infection. Wireless signal transmission will at some stage address that particular issue.

A more truculent problem may be the observed tendency for the ability of microelectrodes recording from neurons to fall off over time, for reasons unknown. Individual responses to the implants also vary sharply: a second patient in Donoghue's experiment was unable to achieve the same degree of control as the first. And there are further issues to be faced: if scientists were to succeed in restoring limb function, they would have to work out how the body tells the brain where its limbs are positioned in space, through a littleunderstood sense called proprioception (see page 125).

A sign of how far the science of neuroprosthetics has come is that most of these difficulties are now engineering challenges, rather than problems of principle. In applauding this valuable
"The hope is to give paralysed patients greater ability to interact with their environments." work, it is worth noting that it was made possible by two of the bêtes noires of modern biology: commercial interests and animal research.

Donoghue's team was supported by a private company in Massachusetts - Cyberkinetics Neurotechnology Systems - of which Donoghue is founder, director and chief scientific officer. Such hearty involvement by commercial interests is unusual, at least in this area of neuroscience. Experience from other scientific disciplines suggests that this route will lead to new challenges, particularly in the free and open dissemination of data (see Nature 442, 1; 2006). However, these issues can be addressed, and they should not stop companies such as Cyberkinetics taking this knowledge from the laboratory to the clinic.

Both papers also owe a great deal to primate research, including the sort of curiosity-driven research most fiercely denounced by the animal-rights movement. Although some work can be done in rats, the best model of the human motor cortex is that of a monkey, the rhesus macaque. Scientists have spent years learning how the macaque's brain works, without necessarily planning to develop neuroprostheses. Experiments on primates will always be contentious, but this work shows why the immediate utility of a particular strand of research should not be the sole factor in determining whether it is ethical for it to proceed.

Although the videos of these latest experiments may garner a great deal of well-deserved attention, it is worth recalling that work in neuroprosthetics is by no means confined to spheres imprinted on the public's collective imagination by science fiction. Steve Austin was rebuilt to have enhanced strength, speed and vision; to be "better than he was before". The idea of giving people superhuman powers greatly appeals to the popular imagination. But in the real world, using neuroprosthetics to give patients control over all the less glamorous things we take for granted will be more important. 